\title{
A Characterization of the Angle Defect and the Euler Characteristic in Dimension 2
}

\author{
Ethan D. Bloch
}

Received: 3 January 2008 / Revised: 18 May 2008 / Accepted: 25 May 2008 /

Published online: 15 July 2008

(C) Springer Science+Business Media, LLC 2008

\begin{abstract}
The angle defect, which is the standard way to measure the curvatures at the vertices of polyhedral surfaces, goes back at least as far as Descartes. Although the angle defect has been widely studied, there does not appear to be in the literature an axiomatic characterization of the angle defect. In this paper a characterization of the angle defect for simplicial surfaces is given, and it is shown that variants of the same characterization work for two known approaches to generalizing the angle defect to arbitrary 2-dimensional simplicial complexes. Simultaneously, a characterization of the Euler characteristic for 2-dimensional simplicial complexes is given in terms of being geometrically locally determined.
\end{abstract}

Keywords Angle defect · Euler characteristic - Simplicial complex · Curvature · Descartes-Gauss-Bonnet Theorem

\section{Introduction}

This paper is concerned with two related questions. The first issue concerns the curvature at a vertex $v$ of a triangulated polyhedral surface $M$, which is given by $d(v, M)=2 \pi-\sum_{\alpha \ni v} \alpha$, where the $\alpha$ are the angles at $v$ of the triangles containing $v$. This curvature function, which we refer to as the "classical angle defect," goes back at least as far as Descartes (see [9]). The classical angle defect satisfies various properties one would expect a curvature function on polyhedra to satisfy. For example, the angle defect is locally defined; it is invariant under simplicial local isometries (that is, functions that preserve the lengths of edges); it is zero at a vertex that has a flat star; it is invariant under subdivision; and it satisfies the polyhedral DescartesGauss-Bonnet Theorem, which says $\sum_{v \in M} d(v, M)=2 \pi \chi(M)$, where the summation is over all the vertices of $M$, and where $\chi(M)$ is the Euler characteristic of $M$.

E.D. Bloch $(\bowtie)$

Bard College, Annandale-on-Hudson, NY 12504, USA

e-mail: bloch@bard.edu 
(We refer to this theorem as the "Descartes-Gauss-Bonnet Theorem" rather than just "Descartes' Theorem" because Descartes' version was for convex polytopes only and did not explicitly involve the Euler characteristic for 2-dimensional polyhedra; there appears to be some dispute in the literature as to whether or not Descartes was implicitly aware of the Euler characteristic.)

The angle defect (also known as the angle deficiency), and related constructs involving sums of angles in polyhedra, have been widely studied, both in the classical situation, as well as in higher dimensions. It has been studied in the case of convex polytopes from a combinatorial approach, for example in [21] and [14]; more generally, for the wider study of angle sums in convex polytopes, see for example [13, Chap. 14], [18-20]. In [15] a Descartes-Gauss-Bonnet Theorem is proved for the angle defect in polytopes with underlying spaces that are manifolds. This approach has been generalized to arbitrary simplicial complexes in [3] and further studied in $[4,5]$. A different approach to generalizing the classical angle defect has been studied extensively from a differential geometric point of view; see, among others, [1, 6-8, $22,23]$. One treatment of curvature of polyhedra that has some of the advantages of all the approaches cited above is in [2], which uses curvatures functions based on critical points (similarly to [1]), but this time using projection maps $\mathbb{R}^{n} \rightarrow \mathbb{R}^{m}$, which leads to curvature functions related to the Grassmann angles of [14] located at all simplices, and which directly generalizes the approach of [1] et al.; moreover, an angle defect type formula for curvature is obtained using projection maps $\mathbb{R}^{n} \rightarrow \mathbb{R}^{n-1}$. (The angle defect and its generalizations treated in the above references, and which we discuss in this note, are geometric in nature, depending upon the measurement of angles; we will not be discussing the combinatorial approach to curvature of simplicial complexes found in [11].)

Although the angle defect has been widely studied, there does not appear to be in the literature an axiomatic characterization of the angle defect. Such a characterization would be useful not only for gaining insight into the angle defect, but also to help distinguish between different generalizations of the classical angle defect to arbitrary polyhedra. In the present paper we give a characterization of the classical angle defect for simplicial surfaces and show that variants of the same characterization work for two approaches to generalizing the classical angle defect to arbitrary 2-dimensional simplicial complexes, as found in [3] on the one hand, and in [1] et al. on the other.

The second issue we discuss concerns the Euler characteristic, which is intimately connected to the classical angle defect by the Descartes-Gauss-Bonnet Theorem. In [10], which follows [17], it is shown that the Euler characteristic is the unique locally determined numerical invariant of finite simplicial complexes that assigns the same number to every cone, where in this context simplicial complexes are considered to be the same if they are combinatorially equivalent. A real-valued function $\rho$ on the set of all finite simplicial complexes is "locally determined" if there is another real-valued function $h$ on the set of all finite simplicial complexes such that for each simplicial complex $K$, we have $\rho(K)=\sum_{v \in K} h(\operatorname{link}(v, K))$, where $\operatorname{link}(v, K)$ denotes the link of $v$ in $K$, and where the summation is over all the vertices of $K$. This last condition certainly has some resemblance to the Descartes-Gauss-Bonnet Theorem, but there is a substantial difference between the results of [17] and [10] on the one hand, and the Descartes-Gauss-Bonnet Theorem on the other: the nature 
of being locally determined in [17] and [10] is purely combinatorial, whereas in the Descartes-Gauss-Bonnet Theorem the Euler characteristic is locally determined by a geometric quantity, namely the classical angle defect, which depends not only on the combinatorial nature of the link of each vertex, but on the geometry of the embedding of the star of each vertex. One might therefore think of the results of [17] and [10] as characterizing the Euler characteristic among those functions that are "combinatorially locally determined." In the present paper we will give an analogous characterization of the Euler characteristic in the 2-dimensional case among those functions that are "geometrically locally determined," a term that will be defined below. Not surprisingly, our characterizations of both the angle defect and the Euler characteristic in dimension 2 are simply different ways of looking at the same result.

\section{Statement of Results}

We start with some assumptions, notation, and definitions. Throughout this paper all simplicial complexes will be 2-dimensional, finite, and embedded in Euclidean space. Different embeddings of combinatorially equivalent simplicial complexes will be considered as different simplicial complexes (in contrast to [17] and [10], where the approach is combinatorial rather than geometric). Embedded simplicial complexes have angles that can be measured, and hence suit our geometric approach more than abstract simplicial complexes. We use the term simplicial surface to mean a 2dimensional simplicial complex the underlying space of which is a compact surface without boundary.

Let $K$ be a simplicial complex. We let $|K|$ denote the underlying space of $K$, let $K^{(0)}$ denote the set of vertices of $K$, and let $f_{i}(K)$ denote the number of $i$-simplices of $K$ for each possible value of $i$. Let $\langle v, w\rangle$ denote the 1 -simplex in $K$ with vertices $v$ and $w$, and we let $\mathcal{O}(v, w)$ denote the number of 2-simplices of $K$ that contain $\langle v, w\rangle$. If $\sigma \in K$, let $\operatorname{link}(\sigma, K)$ and $\operatorname{star}(\sigma, K)$ denote the link and star of $\sigma$ in $K$ respectively. (For basic definitions in PL topology, see for example [12, vol. I] and

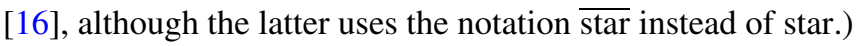

For the sake of convenience, we adopt the convention that all angles in 2-simplices are normalized so that the circumference of the unit circle is 1. For any 2-simplex $\sigma^{2}$ in Euclidean space and any vertex $v$ of $\sigma^{2}$, we let $\alpha\left(v, \sigma^{2}\right)$ denote the (interior) angle in $\sigma^{2}$ at $v$, where by normalization such an angle is always a number in $\left[0, \frac{1}{2}\right]$. Hence the $2 \pi$ will drop out of our statement of the Descartes-Gauss-Bonnet Theorem.

The Euler characteristic is a real-valued function on the set of all 2-dimensional simplicial complexes. We can restrict the Euler characteristic to any set of 2dimensional simplicial complexes, and we will consider other real-valued functions on sets of 2-dimensional simplicial complexes. The classical angle defect is a different type of function, as we now define.

Definition Let $\mathcal{T}$ be a set of 2-dimensional simplicial complexes. A real-valued vertex-supported function on $\mathcal{T}$ is a function $\phi$ that assigns to every 2-dimensional simplicial complex $K \in \mathcal{T}$ and every vertex $v \in K^{(0)}$, a real number $\phi(v, K)$. 
We will use various properties of real-valued vertex-supported functions. These properties, defined below, are all satisfied by the classical angle defect, as can be verified easily. The first property involves subdivision.

Definition Let $\mathcal{T}$ be a set of 2-dimensional simplicial complexes, and let $\phi$ be a realvalued vertex-supported function on $\mathcal{T}$. The function $\phi$ is invariant under subdivision if the following condition holds. If $K, J \in \mathcal{T}$, where $J$ is a subdivision of $K$, and if $v \in K^{(0)}$, then $\phi(v, K)=\phi(v, J)$.

For our next property, which involves isometries, we need the following terminology. Let $K$ and $L$ be 2-dimensional simplicial complexes. We say that $K$ and $L$ are simplicially isometric if there is a simplicial homeomorphism $|K| \rightarrow|L|$ that preserves the lengths of edges; such a map is called a simplicial isometry. Moreover, let $v \in K^{(0)}$, and let $w \in L^{(0)}$. We say that $\operatorname{star}(v, K)$ and $\operatorname{star}(w, L)$ are simplicially isometric if there is a simplicial isometry $|\operatorname{star}(v, K)| \rightarrow|\operatorname{star}(w, L)|$ that takes $v$ to $w$; any simplicial isometry between $\operatorname{star}(v, K)$ and $\operatorname{star}(w, L)$ will always be assumed to take $v$ to $w$.

Definition Let $\mathcal{T}$ be a set of 2-dimensional simplicial complexes, and let $\phi$ be a realvalued vertex-supported function on $\mathcal{T}$. The function $\phi$ is invariant under simplicial isometries of stars if the following condition holds. If $K, L \in \mathcal{T}$, if $v \in K^{(0)}$ and $w \in L^{(0)}$, and if $\operatorname{star}(v, K)$ and $\operatorname{star}(w, L)$ are simplicially isometric, then $\phi(v, K)=$ $\phi(w, L)$.

Our next property involves continuity. Suppose that $K$ and $\left\{K_{n}\right\}_{n=1}^{\infty}$ are combinatorially equivalent 2-dimensional simplicial complexes and all are embedded in the same Euclidean space. We can think of all these 2-dimensional simplicial complexes as embeddings of the same abstract simplicial complex. We write $\lim _{n \rightarrow \infty} K_{n}=K$ to denote pointwise convergence of these embeddings; it suffices to verify convergence at the vertices of the abstract simplicial complex.

Definition Let $\mathcal{T}$ be a set of 2-dimensional simplicial complexes, and let $\phi$ be a realvalued vertex-supported function on $\mathcal{T}$. The function $\phi$ is continuous if the following condition holds. Let $K$ and $\left\{K_{n}\right\}_{n=1}^{\infty}$ be combinatorially equivalent 2-dimensional simplicial complexes in $\mathcal{T}$, all embedded in the same Euclidean space. Suppose $\lim _{n \rightarrow \infty} K_{n}=K$. If $v \in K^{(0)}$, and if the corresponding vertices of each $K_{n}$ is labeled $v_{n}$, then $\lim _{n \rightarrow \infty} \phi\left(v_{n}, K_{n}\right)=\phi(v, K)$.

Our final property is an analog of the Descartes-Gauss-Bonnet Theorem.

Definition Let $\mathcal{T}$ be a set of 2-dimensional simplicial complexes, let $\phi$ be a realvalued vertex-supported function on $\mathcal{T}$, and let $\Lambda$ be a real-valued function on $\mathcal{T}$. The function $\phi$ satisfies the Descartes-Gauss-Bonnet Theorem with respect to $\Lambda$ if the following condition holds. If $K \in \mathcal{T}$, then $\sum_{v \in K} \phi(v, K)=\Lambda(K)$, where the sum is over all the vertices of $K$. 
We now state our results, which take place in two contexts, namely sets of simplicial surfaces on the one hand, and sets of finite 2-dimensional simplicial complexes on the other hand. We first consider things from the point of view of the classical angle defect and its generalizations; shortly we will turn to the point of view of the Euler characteristic and its generalizations. For simplicial surfaces, we have the following characterization of the classical angle defect.

Theorem 2.1 Let $\phi$ be a real-valued vertex-supported function on the set of all simplicial surfaces. Then $\phi$ is the classical angle defect iff $\phi$ is invariant under simplicial isometries of stars and under subdivision, is continuous, and satisfies the DescartesGauss-Bonnet Theorem with respect to the Euler characteristic.

We will deduce Theorem 2.1 as an immediate corollary to Theorem 2.4 below.

It would be interesting to know whether the four hypotheses in Theorem 2.1 are all needed to characterize the classical angle defect. We do not have a complete answer to this question, though the following examples provide a partial answer.

\section{Example 2.2}

(1) It is clear that the Descartes-Gauss-Bonnet Theorem cannot be dropped in Theorem 2.1 , because the constantly zero real-valued vertex-supported function on the set of all simplicial surfaces satisfies the three other criteria of the theorem.

(2) If $K$ is a simplicial surface, and if $v \in K^{(0)}$, let $E(v, K)$ denote the number of edges of $K$ that contain $v$. Define the real-valued vertex-supported function $\psi$ on the set of all simplicial surfaces by letting $\psi(v, K)=1-\frac{1}{6} E(v, K)$ for any simplicial surface $K$ and any vertex $v$ of $K$. It is evident that $\psi$ is invariant under simplicial isometries of stars and is continuous, and it can also be verified that $\psi$ satisfies the Descartes-Gauss-Bonnet Theorem with respect to the Euler characteristic (using the fact that $\sum_{v \in K} E(v, K)=2 f_{1}(K)=3 f_{2}(K)$, where the summation is over all vertices of $K)$. However, it is evident that $\psi$ is not invariant under subdivision, and therefore the invariance under subdivision criterion cannot be dropped from Theorem 2.1.

(3) Define the real-valued vertex-supported function $\mu$ on the set of all simplicial surfaces as follows. Let $K$ be a simplicial surface. If $\chi(K)=0$, let $\mu(v, K)=0$ for all vertices $v$ of $K$. Now suppose that $\chi(K) \neq 0$. Let $n(K)$ denote the number of vertices $v$ of $K$ for which the sum of the angles at $v$ is not equal to 1 ; observe that $n(K) \neq 0$ because of the Descartes-Gauss-Bonnet Theorem for the classical angle defect. Let

$$
\mu(v, K)= \begin{cases}\chi(K) / n(K), & \text { if the sum of the angle containing } v \text { is not } 1, \\ 0, & \text { otherwise, }\end{cases}
$$

for every vertex $v$ of $K$. It is clear that $\mu$ satisfies the Descartes-Gauss-Bonnet Theorem with respect to the Euler characteristic, and it can also be seen that $\mu$ is invariant under subdivision (because any new vertex in a subdivision of a 2-dimensional simplicial complex $K$ is in the relative interior of a 1-simplex or a 2 -simplex of $K$, and hence has angle sum equal to 1 ). We leave it to the 
reader to verify that $\mu$ is not invariant under simplicial isometries of stars and is not continuous. Hence, these last two criteria cannot both be dropped from Theorem 2.1.

Theorem 2.1 holds unchanged for the class of all 2-dimensional simplicial pseudomanifolds (without boundary), and we omit the details. The situation becomes more interesting when we go beyond pseudomanifolds and look at the class of all 2dimensional simplicial complexes, because for non-pseudomanifolds, there are (at least) two generalizations of the classical angle defect, both of which are equal to the classical angle defect when restricted to pseudomanifolds.

Both of these generalizations of the classical angle defect work in all dimensions. The first of these generalizations, which we refer to as "standard curvature," is discussed, among others, in [1, 6-8, 22, 23]. It is very simple to define (though it does not directly resemble the classical angle defect), and it satisfies our four properties. In all dimensions, this type of curvature is concentrated at the vertices of simplicial complexes.

The second generalization of the classical angle defect, which we refer to as the "generalized angle defect," was defined in [3] and further studied in [4, 5]. This type of curvature, which more closely resembles the classical angle defect than standard curvature, is a generalization of the higher-dimensional angle defect for convex polytopes and manifolds studied, among others, in [14, 15, 21]. In dimensions higher than 2, the generalized angle defect is not concentrated at the vertices of simplicial complexes, but rather is defined for each simplex of codimension at least 2 . (A word on our terminology. In order to compare our approach with standard curvature, we originally somewhat artificially concentrated our curvature at the vertices in [3, Sect. 3] and called it "stratified curvature." In [3, Sect. 4] we took the more natural approach that we are using at present and referred to this approach by the unfortunate name "modified stratified curvature," which misses the point that in this approach we are really working with a pure angle defect. Hence, in the present paper, we will use the better name "generalized angle defect," which we also use in $[4,5]$. A detailed comparison of standard curvature with both stratified curvature and the generalized angle defect may be found in [3, Sect. 4].)

It turns out that in the present paper we will rarely need the actual definition of standard curvature and we will never need the definition of the generalized angle defect-what we use regularly are the properties of these types of curvature. Like standard curvature, the generalized angle defect is invariant under simplicial isometries of stars and under subdivision, and it is continuous. It also satisfies the Descartes-Gauss-Bonnet Theorem, though not with respect to the Euler characteristic but with respect to a variant of the Euler characteristic, called the stratified Euler characteristic. See [3] for the definition of the stratified Euler characteristic and a proof of the Descartes-Gauss-Bonnet Theorem for the generalized angle defect.

The following theorem characterizes both these types of curvatures on the set of all 2-dimensional simplicial complexes.

Theorem 2.3 Let $\phi$ be a real-valued vertex-supported function on the set of all 2dimensional simplicial complexes. Then $\phi$ is standard curvature (respectively the generalized angle defect) iff $\phi$ is invariant under simplicial isometries of stars and under 
subdivision, is continuous, and satisfies the Descartes-Gauss-Bonnet Theorem with respect to the Euler characteristic (respectively the stratified Euler characteristic).

Theorem 2.3 sheds light on how similar standard curvature and the generalized angle defect are in dimension 2. These two types of curvature are less similar in higher dimensions, because of the fact that standard curvature is concentrated at vertices and the generalized angle defect is not. A better understanding of the difference between these two types of curvature awaits characterizations of standard curvature and the generalized angle defect in higher dimensions. Unfortunately, our proof of Theorem 2.3 (which is really a corollary to Theorem 2.7 stated below) does not generalize beyond the 2-dimensional case. It would be interesting to know whether the higherdimensional analogs of our results are nonetheless true, using a different method of proof.

Also, we note that Theorem 2.3 does not imply Theorem 2.1, because more is being assumed about $\phi$ in Theorem 2.3 than in Theorem 2.1.

We now turn our attention to the point of view of the Euler characteristic and its generalizations. The following definition gives our geometric analog of the notion of being locally determined (as discussed in [10, 17]).

Definition Let $\mathcal{T}$ be a set of 2-dimensional simplicial complexes, and let $\Lambda$ be a real-valued function on $\mathcal{T}$. The function $\Lambda$ is geometrically locally determined if there is a real-valued vertex-supported function $\phi$ on $\mathcal{T}$ such that $\phi$ is invariant under simplicial isometries of stars and under subdivision, is continuous, and satisfies the Descartes-Gauss-Bonnet Theorem with respect to $\Lambda$. If we need to specify $\phi$, we will say that $\Lambda$ is geometrically locally determined by $\phi$.

We use the adverb "geometrically" in the above definition because of the requirement of invariance under subdivision, which is a very plausible requirement if one thinks of the curvature of polyhedra geometrically, but which does not work well from a combinatorial point of view. Recall the combinatorial approach of [17] and [10], in which a real-valued function $\rho$ on the set of all simplicial complexes is locally determined if there is another real-valued function $h$ on the set of all simplicial complexes such that for each simplicial complex $K$, we have $\rho(K)=\sum_{v \in K} h(\operatorname{link}(v, K))$, where the summation is over all the vertices of $K$; in this context, the functions $\rho$ and $h$ depend only upon combinatorial equivalence, not upon the particular embedding of a simplicial complex. We can then think of $h(\operatorname{link}(v, K))$ as a real-valued vertex-supported function on the set of all simplicial complexes. If $h(\operatorname{link}(v, K))$ is to be invariant under subdivision, then it would be necessary for $h$ to be invariant under subdivision. However, let $K$ be a simplicial surface, and let $L$ be the subdivision of $K$ obtained by adding a single vertex $w$ in the relative interior of a 2-simplex of $K$, and then starring on $w$. Up to combinatorial equivalence, the link of every vertex of $L$ other than $w$ is a subdivision of the link of the same vertex in $K$. Suppose that $\rho$ is invariant under homotopy equivalence (as in [17] and [10]) and that $h$ is invariant under subdivision. Then $\rho(K)=\rho(L)$ and $h(\operatorname{link}(v, K))=h(\operatorname{link}(v, L))$ for all $v \in K^{(0)}$. It follows that $h(\operatorname{link}(w, L))=0$. Observe that $\operatorname{link}(w, L)$ is a triangle and that the link of any vertex in any simplicial surface is combinatorially equivalent 
to a subdivision of a triangle. Hence, the invariance of $h$ under subdivision implies that $h(\operatorname{link}(v, K))=0$ for all $v \in K^{(0)}$, which means that $\rho(K)=0$. Therefore $\rho$ is constantly 0 on the set of all simplicial surfaces, which means that $\rho$ is not very interesting, and in particular it cannot be the Euler characteristic. Hence, invariance under subdivision rules out a purely combinatorial approach, as in [17] and [10].

Not every real-valued function on a set of 2-dimensional simplicial complexes is geometrically locally determined. For example, it can be verified that the function $\Lambda$ on the set of all 2-dimensional simplicial complexes defined by $\Lambda(K)=f_{2}(K)$ is not geometrically locally determined; we omit the details. Hence, we will restrict our attention to those real-valued functions that are well behaved in some appropriate way. In [10], as seen in the title of that paper, the condition of being constant on the set of cones is used; this condition is weaker than the condition of being a homotopy invariant, which is used in [17]. Because the stratified Euler characteristic of [3, Sect. 2] is not a homotopy invariant (though it is a homeomorphism invariant), we adopt the approach of [10] and will consider real-valued functions that are constant on a number of different sets of 2-dimensional simplicial complexes.

We will use the following standard terminology. Let $D$ be a simplicial disk in $\mathbb{R}^{2}$. A pyramid on $D$ is the simplicial surface obtained by coning on $D$ from a point in $\mathbb{R}^{3}$ (called the apex of the pyramid) that is not in $\mathbb{R}^{2}$ and then taking the boundary. Let $R$ be a polygonal disk in $\mathbb{R}^{2}$ (not necessarily subdivided into simplices). A bipyramid on $R$ is the simplicial surface obtained by suspending $R$ from two points in $\mathbb{R}^{3}$ (called the apices of the bipyramid) that are in different half-space components of the complement of $\mathbb{R}^{2}$ and then taking the boundary. We also need the following less standard terms.

Definition A planar fan is a simplicial disk in $\mathbb{R}^{2}$ with no interior vertices that is triangulated as a cone from one of its boundary vertices. Let $n \in \mathbb{Z}$ be such that $n \geq 0$. An $n$-flap with end-vertices $v$ and $w$ is a 2-dimensional simplicial complex $K$ containing vertices $v$ and $w$ such that the following conditions hold: (1) $\langle v, w\rangle$ is an edge of $K$; (2) $K$ has precisely $n$ 2-simplices, each of which has $\langle v, w\rangle$ as an edge; and (3) $K$ has no edges other than the 1 -faces of these $n 2$-simplices.

A 0-flap is a single edge, and a 1-flap is a single 2-simplex. It is straightforward to verify that a 2-dimensional simplicial complex $L$ is an $n$-flap with end-vertices $v$ and $w$ iff $L=\operatorname{star}(v, L)=\operatorname{star}(w, L)$. Also, if $K$ is a 2-dimensional simplicial complex and $v \in K^{(0)}$, and if $N=\operatorname{star}(v, K)$, then for any vertex $x$ of $\operatorname{link}(v, K)$, it is seen that $\operatorname{star}(x, N)$ is a $\mathcal{O}(v, x)$-flap.

As mentioned above, we will consider real-valued functions that are constant on various sets of 2-dimensional simplicial complexes. In particular, if $\Lambda$ is a real-valued function, we will consider the case where $\Lambda$ is constant on the set of all planar fans, and we will write $\Lambda$ (fan); the case where $\Lambda$ is constant on the set of all n-flaps, and we will write $\Lambda$ ( $n$-flap), for each $n \in \mathbb{N}$; the case where $\Lambda$ is constant on the set of all pyramids and bipyramids, and we will write $\Lambda$ (pyramid); and the case where $\Lambda$ is constant on the set of all cones, and we will write $\Lambda$ (cone).

For simplicial surfaces, our main technical result is the following theorem. 
Theorem 2.4 Let $\mathcal{S}$ be a set of simplicial surfaces that contains all pyramids and bipyramids. Let $\Lambda$ be a real-valued function on $\mathcal{S}$. Suppose that $\Lambda$ is geometrically locally determined by a real-valued vertex-supported function $\phi$ on $\mathcal{S}$ and that $\Lambda$ is constant on the set of all pyramids and bipyramids. If $K \in \mathcal{S}$ and $v \in K^{(0)}$, then

$$
\phi(v, K)=\frac{1}{2} \Lambda(\text { pyramid })\left[1-\sum_{\sigma^{2} \ni v} \alpha\left(v, \sigma^{2}\right)\right],
$$

where the summation is over all 2-simplices of $K$ containing $v$.

The proof of Theorem 2.4 will be given in Sect. 3. It is straightforward to see that Theorem 2.1 follows immediately from Theorem 2.4. Moreover, we have the following corollaries to Theorem 2.4, which characterize the Euler characteristic on sets of simplicial surfaces. The first of these corollaries follows immediately from Theorem 2.4 because of the Descartes-Gauss-Bonnet Theorem for the classical angle defect, and the second corollary follows from the first.

Corollary 2.5 Let $\mathcal{S}$ be a set of simplicial surfaces that contains all pyramids and bipyramids. Let $\Lambda$ be a real-valued function on $\mathcal{S}$. Suppose that $\Lambda$ is geometrically locally determined and is constant on the set of all pyramids and bipyramids. Then $\Lambda$ equals the Euler characteristic multiplied by $\frac{1}{2} \Lambda$ (pyramid).

Corollary 2.6 Let $\mathcal{S}$ be a set of simplicial surfaces that contains all pyramids and bipyramids. The Euler characteristic is the unique real-valued function on $\mathcal{S}$ that is geometrically locally determined and has value 2 on all pyramids and bipyramids.

We now turn to analogs of the above results for more general sets of 2-dimensional simplicial complexes, starting with the following definition, which is based on [10].

Definition Let $\mathcal{T}$ be a set of 2-dimensional simplicial complexes. The set $\mathcal{T}$ is starclosed if for every $K \in \mathcal{T}$ and every vertex $v$ of $K$, we have $\operatorname{star}(v, K) \in \mathcal{T}$.

Clearly, the set of all finite 2-dimensional simplicial complexes is star-closed.

We can now state an analog for arbitrary 2-dimensional simplicial complexes of Theorem 2.4.

Theorem 2.7 Let $\mathcal{T}$ be a star-closed set of 2-dimensional simplicial complexes that contains all planar fans. Let $\Lambda$ be a real-valued function on $\mathcal{T}$. Suppose that $\Lambda$ is geometrically locally determined by a real-valued vertex-supported function $\phi$ on $\mathcal{T}$, that $\Lambda$ is constant on the set of all planar fans, and that for each $n \geq 0$, the function $\Lambda$ is constant on the set of all $n$-flaps in $\mathcal{T}$. If $K \in \mathcal{T}$ and $v \in K^{(0)}$, then

$$
\begin{aligned}
\phi(v, K)= & \Lambda(\operatorname{star}(v, K))-\frac{1}{2} \sum_{w \in \operatorname{link}(v, K)} \Lambda(\mathcal{O}(v, w)-\text { flap }) \\
& +\frac{1}{2} \Lambda(\text { fan }) f_{1}(\operatorname{link}(v, K))-\Lambda(\text { fan }) \sum_{\sigma^{2} \ni v} \alpha\left(v, \sigma^{2}\right),
\end{aligned}
$$


where the first summation is over all vertices $w$ of $\operatorname{link}(v, K)$, and the second summation is over all 2-simplices of $K$ containing $v$.

One could view Theorem 2.7 as a geometric, 2-dimensional analog of the uniqueness stated in [17, Theorem B]. The following result is an immediate consequence of Theorem 2.7.

Corollary 2.8 Let $\mathcal{T}$ be a star-closed set of 2-dimensional simplicial complexes that contains all planar fans. Let $\Lambda$ be a real-valued function on $\mathcal{T}$. Suppose that $\Lambda$ is geometrically locally determined, that $\Lambda$ is constant on the set of all planar fans, and that for each $n \geq 0$, the function $\Lambda$ is constant on the set of all $n$-flaps in $\mathcal{T}$. Then there is a unique real-valued vertex-supported function $\phi$ on $\mathcal{T}$ such that $\Lambda$ is geometrically locally determined by $\phi$.

We note that Theorem 2.7 implies that any real-valued function $\Lambda$ that is geometrically locally determined and satisfies appropriate hypotheses, is not only geometrically locally determined by a unique real-valued vertex-supported function $\phi$, but that such $\phi$ is similar to an angle defect, in that the first three terms in the right-hand side of (2) do not depend upon the particular embedding of $K$ in Euclidean space, and hence the right-hand side of (2) has the form of a measure of flatness (which is 1 in the case of simplicial surfaces and in general depends only upon the topology of a neighborhood of $v$ ) minus the sum of the angles at $v$ (once the term $\Lambda$ (fan) has been factored out).

It is straightforward to see that Theorem 2.3 follows immediately from Corollary 2.8 .

The following two corollaries to Theorem 2.7 characterize the Euler characteristic on sets of 2-dimensional simplicial complexes. The first of these corollaries will be proved in Sect. 3, and the second corollary follows from the first.

Corollary 2.9 Let $\mathcal{T}$ be a star-closed set of 2-dimensional simplicial complexes that contains all planar fans. Let $\Lambda$ be a real-valued function on $\mathcal{T}$. Suppose that $\Lambda$ is geometrically locally determined and that $\Lambda$ is constant on the set of all cones in $\mathcal{T}$. Then $\Lambda$ equals the Euler characteristic multiplied by $\Lambda$ (cone).

Corollary 2.10 Let $\mathcal{T}$ be a star-closed set of 2-dimensional simplicial complexes that contains all planar fans. The Euler characteristic is the unique real-valued function on $\mathcal{T}$ that is geometrically locally determined and has value 1 on all cones in $\mathcal{T}$.

We note that Corollary 2.9, which is our analog of the main result in [17] and [10], neither implies that result nor is implied by it, because the latter does not differentiate between different embeddings of combinatorially equivalent 2-dimensional simplicial complexes, and the former requires invariance under subdivision, which is not required for the latter. Nonetheless, there is a relation between Corollary 2.9 and the main result in [17] and [10], which is seen as follows. As mentioned in [10], the Euler characteristic is locally determined (in the combinatorial sense) by the function $e$ on 
the set of all simplicial complexes defined by

$$
e(M)=1+\sum_{i=0}^{1} \frac{(-1)^{i+1}}{i+2} f_{i}(M)
$$

for all 2-dimensional simplicial complexes $M$. Let $K$ be a 2-dimensional simplicial complex. The meaning of locally determined is that $\chi(K)=\sum_{v \in K} e(\operatorname{link}(v, K))$, where the summation is over all vertices of $K$. Because $K$ is 2-dimensional, then for each $v \in K^{(0)}$, the above formula for $e$ yields $e(\operatorname{link}(v, K))=1-\frac{1}{2} f_{0}(\operatorname{link}(v, K))+$ $\frac{1}{3} f_{1}(\operatorname{link}(v, K))$.

On the other hand, in our present context, the Euler characteristic on the set of all 2-dimensional simplicial complexes is geometrically locally determined by standard curvature, which in turn is computed via exterior angles at vertices of simplices. Let $v \in K^{(0)}$. For any simplex $\eta$ of $K$ of dimension 0,1 , or 2 that has $v$ as a vertex, the exterior angle of $\eta$ at $v$, denoted $\alpha^{*}(v, \eta)$, is computed as follows: if $\eta$ is a 2-simplex, then $\alpha^{*}(v, \eta)=\frac{1}{2}-\alpha(v, \eta)$; if $\eta$ is a 1-simplex, then $\alpha^{*}(v, \eta)=\frac{1}{2}$; if $\eta$ is a 0 -simplex (so that $\sigma=v$ ), then $\alpha^{*}(v, \eta)=1$. The standard curvature of $K$ at $v$ is given by

$$
S(v, K)=\sum_{i=0}^{2}(-1)^{i} \sum_{\eta^{i} \ni v} \alpha^{*}\left(v, \eta^{i}\right),
$$

where the inner summation is over all $i$-simplices of $K$ containing $v$; see [1], for example.

Now suppose that $K$ has been embedded in Euclidean space so that all 2-simplices are equilateral triangles (such an embedding is always possible, as a subcomplex of a unit $p$-simplex in $\mathbb{R}^{p}$ for sufficiently large $p$ ). If $\eta$ is a 2 -simplex of $K$, then $\alpha^{*}(v, \eta)=\frac{1}{2}-\alpha(v, \eta)=\frac{1}{2}-\frac{1}{6}=\frac{1}{3}$. Therefore

$$
\begin{aligned}
S(v, K) & =\sum_{i=0}^{2}(-1)^{i} \sum_{\eta^{i} \ni v} \alpha^{*}\left(v, \eta^{i}\right) \\
& =\alpha^{*}(v, v)-\sum_{\eta^{1} \ni v} \alpha^{*}\left(v, \eta^{1}\right)+\sum_{\eta^{2} \ni v} \alpha^{*}\left(v, \eta^{2}\right) \\
& =1-\sum_{\eta^{1} \ni v} \frac{1}{2}+\sum_{\eta^{2} \ni v} \frac{1}{3} \\
& =1-\frac{1}{2} f_{0}(\operatorname{link}(v, K))+\frac{1}{3} f_{1}(\operatorname{link}(v, K)) .
\end{aligned}
$$

Hence, for this particular embedding of $K$, we have $S(v, K)=e(\operatorname{link}(v, K))$, which means that the combinatorial and geometric types of being locally determined coincide. 


\section{Proofs}

We remind the reader that throughout this paper all simplicial complexes will be 2dimensional, finite, and embedded in Euclidean space; different embeddings of combinatorially equivalent simplicial complexes will be considered as different simplicial complexes.

In this section we will prove Theorem 2.7, Corollary 2.9, and Theorem 2.4.

Proof of Theorem 2.7 We start with the following preliminary observation. Let $K$ and $L$ be 2-dimensional simplicial complexes, let $v \in K^{(0)}$, and let $u \in L^{(0)}$. Suppose that $\operatorname{link}(v, K)$ and $\operatorname{link}(u, L)$ are both polygonal arcs and that the sum of the angles at $v$ equals the sum of the angles at $u$. Then there are subdivisions $K^{\prime}$ and $L^{\prime}$ of $K$ and $L$ respectively such that $\operatorname{star}\left(v, K^{\prime}\right)$ and $\operatorname{star}\left(u, L^{\prime}\right)$ are simplicially isometric. It then follows from the invariance of $\phi$ under subdivision and under simplicial isometries of stars that $\phi(v, K)=\phi\left(v, K^{\prime}\right)=\phi\left(u, L^{\prime}\right)=\phi(u, L)$.

Our proof has a number of steps, first looking at some special cases and then proving the result in general in the last step. In each step, we will let $K$ be a 2-dimensional simplicial complex, and we will let $v \in K^{(0)}$, where $v$ is subject to certain stated conditions; we will find a formula for $\phi(v, K)$ in the given case. The arguments in many of the steps are similar to each other, and we will omit some of the details.

Step 1 Suppose that $\operatorname{link}(v, K)$ is a polygonal arc, and that the sum of the angles at $v$ is a number $\kappa$ that has the form $\kappa=\frac{n-2}{2 n}$ for some $n \in \mathbb{N}$ such that $n \geq 3$. We will show that $\phi(v, K)=\Lambda(\mathrm{fan})\left[\frac{1}{2}-\kappa\right]$. (It can be verified that this last equation is a special case of (2), though we will not need this fact.)

Let $S$ be a disk in $\mathbb{R}^{2}$, the boundary of which is a regular polygon with $n$ vertices, say $a_{1}, \ldots, a_{n}$. The angle at each $a_{i}$ equals $\frac{n-2}{2 n}$ (recall that we have normalized angles so that a complete circle has angle 1 ). The disk $S$ can be triangulated as a planar fan. Then the link of each $a_{i}$ is a polygonal arc, and the sum of the angles at each $a_{i}$ is $\frac{n-2}{2 n}$. By our preliminary observation, we know that all the $\phi\left(a_{i}, S\right)$ are equal to each other and to $\phi(v, K)$. Applying the Descartes-Gauss-Bonnet Theorem to the disk $S$, we deduce that $\sum_{i=1}^{n} \phi\left(a_{i}, S\right)=\Lambda(S)$, and hence $n \phi(v, K)=\Lambda(S)$, which implies $\phi(v, K)=\frac{1}{n} \Lambda($ fan $)$. However, because $\kappa=\frac{n-2}{2 n}$, we have $n=1 /\left(\frac{1}{2}-\kappa\right)$ and we deduce that $\phi(v, K)=\Lambda($ fan $)\left[\frac{1}{2}-\kappa\right]$.

Step 2 Suppose that $\operatorname{link}(v, K)$ is a polygonal arc, and that the sum of the angles at $v$ is a rational number $\delta$ such that $0<\delta<\frac{1}{2}$. We will show that $\phi(v, K)=$ $\Lambda($ fan $)\left[\frac{1}{2}-\delta\right]$.

Suppose $\delta=\frac{p}{q}$ for some $p, q \in \mathbb{N}$. Because $\delta<\frac{1}{2}$, we have $q \geq 3$. Let $D$ be a convex polygonal disk in $\mathbb{R}^{2}$ with $p+3$ vertices, labeled $s, x, t, a_{1}, \ldots, a_{p}$ in order around the boundary of $D$, so that the angles at $s$ and $t$ are $\frac{1}{4}$, the angle at $x$ is $\delta$, and the angle at each $a_{i}$ is $\frac{q-2}{2 q}$. That such a convex polygon exists is due to the fact that all the angles are less than $\frac{1}{2}$, and the sum of the exterior angles is precisely 1 (as can easily be verified). The disk $D$ can be triangulated as a planar fan. Observe that $\frac{1}{4}=\frac{4-2}{2 \cdot 4}$, and hence all of the angles in $D$ other than the angle at $x$ satisfy the 
hypothesis of Step 1. Applying the Descartes-Gauss-Bonnet Theorem to the disk $D$, we see that

$$
\phi(s, D)+\phi(x, D)+\phi(t, D)+\sum_{i=1}^{p} \phi\left(a_{i}, D\right)=\Lambda(D) .
$$

By Step 1 we conclude that

$$
\begin{aligned}
\phi(x, D)= & \Lambda(D)-\phi(s, D)-\phi(t, D)-\sum_{i=1}^{p} \phi\left(a_{i}, D\right) \\
= & \Lambda(\text { fan })-\Lambda(\text { fan })\left[\frac{1}{2}-\frac{1}{4}\right]-\Lambda(\text { fan })\left[\frac{1}{2}-\frac{1}{4}\right] \\
& -\sum_{i=1}^{p} \Lambda(\text { fan })\left[\frac{1}{2}-\frac{q-2}{2 q}\right] \\
= & \Lambda(\text { fan })\left[\frac{1}{2}-\frac{p}{q}\right]=\Lambda(\text { fan })\left[\frac{1}{2}-\delta\right] .
\end{aligned}
$$

Hence $\phi(v, K)=\Lambda($ fan $)\left[\frac{1}{2}-\delta\right]$.

Step 3 Suppose that $\operatorname{link}(v, K)$ is a polygonal arc, and that the sum of the angles at $v$ is a real number $\gamma$ such that $0<\gamma<\frac{1}{2}$. We will show that $\phi(v, K)=\Lambda($ fan $)\left[\frac{1}{2}-\gamma\right]$.

There exists a sequence of positive rational numbers $\left\{\delta_{n}\right\}_{n=1}^{\infty}$ such that $0<\delta_{n}<\frac{1}{2}$ for all $n$ and $\lim _{n \rightarrow \infty} \delta_{n}=\gamma$. Let $T=\langle x, y, z\rangle$ be a triangle in $\mathbb{R}^{2}$ such that the angle at $x$ is $\gamma$. Clearly there is a sequence of triangles $\left\{T_{n}\right\}_{n=1}^{\infty}$ in $\mathbb{R}^{2}$, where for each $n$, we have $T_{n}=\left\langle x_{n}, y_{n}, z_{n}\right\rangle$ with the angle at $x_{n}$ equal to $\delta_{n}$ and such that $\lim _{n \rightarrow \infty} T_{n}=T$, with $\lim _{n \rightarrow \infty} x_{n}=x$. By Step 2 we know that $\phi\left(x_{n}, T_{n}\right)=\Lambda$ (fan) $\left[\frac{1}{2}-\delta_{n}\right]$ for all $n$. It follows from the continuity of $\phi$ that $\phi(x, T)=\lim _{n \rightarrow \infty} \phi\left(x_{n}, T_{n}\right)=$ $\lim _{n \rightarrow \infty} \Lambda(\operatorname{fan})\left[\frac{1}{2}-\delta_{n}\right]=\Lambda($ fan $)\left[\frac{1}{2}-\gamma\right]$. Hence $\phi(v, K)=\Lambda($ fan $)\left[\frac{1}{2}-\gamma\right]$.

Step 4 Suppose that $\operatorname{link}(v, K)$ is a polygonal arc, and that the sum of the angles at $v$ is a real number $\beta$ such that $0<\beta<1$. We will show that $\phi(v, K)=\Lambda($ fan $)\left[\frac{1}{2}-\beta\right]$.

Let $Q$ be a quadrilateral in $\mathbb{R}^{2}$ with vertices $e, b_{1}, b_{2}, b_{3}$, such that the angle at $e$ is $\beta$, and the other three angles are less than $\frac{1}{2}$. The quadrilateral $Q$ can be triangulated as a planar fan. The desired result can be obtained by applying the Descartes-GaussBonnet Theorem to the disk $Q$, solving for $\phi\left(b_{1}, Q\right)$, and then using Step 3 and the fact that the sum of the angles in a quadrilateral is 1 .

Step 5 Suppose that $K$ is an $n$-flap for some $n \geq 0$, and that $v$ is one of the endvertices of $K$. We will show that

$$
\phi(v, K)=\frac{1}{2} \Lambda(n \text {-flap })-\Lambda(\text { fan }) \sum_{\sigma^{2} \ni v} \alpha\left(v, \sigma^{2}\right),
$$


where the summation is over all 2-simplices containing $v$. (Again, it can be verified that (3) is a special case of (2), though we will not need this fact.)

Let $w$ be the other end-vertex of $K$. We have four cases.

Subcase 1 Suppose that $n=0$. Then $K$ consists of a single edge $\langle v, w\rangle$ together with its vertices. Clearly $v$ and $w$ have simplicially isometric stars, and hence $\phi(v, K)=\phi(w, K)$. Applying the Descartes-Gauss-Bonnet Theorem to $\phi$, we deduce that $\phi(v, K)=\frac{1}{2} \Lambda(\langle v, w\rangle)$. Because $\langle v, w\rangle$ is a 0 -flap and $v$ is contained in no 2-simplices, (3) holds in this case.

Subcase 2 Suppose that $n=1$. Then $K$ is a single 2-simplex. Let $\omega$ be the angle at $v$ in $K$. We deduce from Step 3 that $\phi(v, K)=\Lambda(\operatorname{fan})\left[\frac{1}{2}-\omega\right]$. This last equation is a special case of (3), using the fact that a 1-flap is a fan.

Subcase 3 Suppose that $n \geq 2$, and suppose further that $\alpha\left(v, \sigma^{2}\right)<\frac{1}{4}$ for all 2simplices $\sigma^{2}$ of $K$ containing $v$.

First, observe that because $K$ is an $n$-flap, we can find an embedding of $K$ in $\mathbb{R}^{3}$ that is simplicially isometric with $K$. Hence, by the invariance of $\phi$ under simplicial isometries of stars, we may assume without loss of generality that $K$ is in $\mathbb{R}^{3}$.

Choose a plane $\Pi$ in $\mathbb{R}^{3}$ that intersects the relative interior of the edge $\langle v, w\rangle$ and is perpendicular to it, and such $v$ is on one side of $\Pi$, and all the other vertices of $K$ are on the other side (such a plane can be found because of the assumption about the angles at $v$ ). Let $V$ be the intersection of $|K|$ with the closed half-space in $\mathbb{R}^{3}$ that has $\Pi$ as its boundary and contains $v$. Let $V^{\prime}$ be the result of reflecting $V$ in $\Pi$, and let $Y=V \cup V^{\prime}$. Then $Y$ is an $n$-flap, which follows from the assumption about the angles at $v$, together with the choice of $\Pi$. Let $x$ denote the vertex of $Y$ that is the mirror image of $v$, let $\tau_{1}, \ldots, \tau_{n}$ denote the $n 2$-simplices of $Y$, and for each $i \in\{1, \ldots, n\}$, let $d_{i}$ denote the vertex in $\tau_{i}$ that is not $v$ or $x$.

By Step 4, making use of the symmetry of $Y$, we see that $\phi\left(d_{i}, Y\right)=\Lambda($ fan $)\left[\frac{1}{2}-\right.$ $\left.\alpha\left(d_{i}, Y\right)\right]=2 \Lambda(\operatorname{fan}) \alpha\left(v, \tau_{i}\right)$ for each $i \in\{1, \ldots, n\}$. By the invariance of $\phi$ under subdivision and under simplicial isometries of stars we see that $\phi(v, K)=\phi(v, Y)=$ $\phi(x, Y)$. The Descartes-Gauss-Bonnet Theorem applied to $Y$ then implies that

$$
2 \phi(v, Y)+\sum_{i=1}^{n} \phi\left(d_{i}, Y\right)=\Lambda(Y)
$$

and hence that

$$
\begin{aligned}
\phi(v, K) & =\phi(v, Y)=\frac{1}{2}\left\{\Lambda(Y)-\sum_{i=1}^{n} \phi\left(d_{i}, Y\right)\right\} \\
& =\frac{1}{2} \Lambda(n \text {-flap })-\Lambda(\text { fan }) \sum_{i=1}^{n} \alpha\left(v, \tau_{i}\right),
\end{aligned}
$$

which is equivalent to (3). 
Subcase 4 Suppose that $n \geq 2$, but we make no assumptions regarding the angles in $K$. (Note that the argument used in Subcase 3 will not work in the general case, because $Y$ as constructed would not always be an $n$-flap.) As in Subcase 3, we may assume without loss of generality that $K$ is in $\mathbb{R}^{3}$.

We now modify $K$ as follows. Let $\zeta_{1}, \ldots, \zeta_{n}$ denote the $n 2$-simplices in $K$, and for each $i \in\{1, \ldots, n\}$, let $e_{i}$ denote the vertex in $\zeta_{i}$ that is not $v$ or $w$. Let $i \in\{1, \ldots, n\}$. If $\alpha\left(w, \zeta_{i}\right)<\frac{1}{4}$, then we leave $\zeta_{i}$ unchanged. If $\alpha\left(w, \zeta_{i}\right) \geq \frac{1}{4}$, then we modify $\zeta_{i}$ by moving $e_{i}$ along the line segment $\left\langle v, e_{i}\right\rangle$ toward $v$ until $\alpha\left(w, \zeta_{i}\right)$ becomes less than $\frac{1}{4}$. This modification will not change $\alpha\left(v, \zeta_{i}\right)$. After we modify all triangles as needed, we call the new $n$-flap $Z$. Similarly to previous arguments, we note that $\phi(v, K)=\phi(v, Z)$. Observe also that the vertex $w$ in $Z$ satisfies the hypotheses of Subcase 3, and so $\phi(w, Z)$ satisfies (3); the vertex $v$ in $Z$ will not necessarily satisfy the hypotheses of Subcase 3.

By Step 4, for each $i \in\{1, \ldots, n\}$, we have

$$
\phi\left(e_{i}, Z\right)=\Lambda(\operatorname{fan})\left[\frac{1}{2}-\alpha\left(e_{i}, Z\right)\right]=\Lambda(\operatorname{fan})\left[\alpha\left(v, \zeta_{i}\right)+\alpha\left(w, \zeta_{i}\right)\right] .
$$

By applying the Descartes-Gauss-Bonnet Theorem to $Z$ and then solving for $\phi(v, Z)$, we see that

$$
\begin{aligned}
\phi(v, K)= & \phi(v, Z)=\Lambda(Z)-\phi(w, Z)-\sum_{i=1}^{n} \phi\left(e_{i}, Z\right) \\
= & \Lambda(n \text {-flap })-\left\{\frac{1}{2} \Lambda(n \text {-flap })-\Lambda(\text { fan }) \sum_{i=1}^{n} \alpha\left(w, \zeta_{i}\right)\right\} \\
& -\Lambda(\text { fan }) \sum_{i=1}^{n}\left[\alpha\left(v, \zeta_{i}\right)+\alpha\left(w, \zeta_{i}\right)\right],
\end{aligned}
$$

which can be rearranged to obtain (3).

Step 6 We now prove our theorem. Suppose that $K$ is a 2-dimensional simplicial complexand that $v \in K^{(0)}$.

Let $N=\operatorname{star}(v, K)$. Clearly $\operatorname{link}(v, K)=\operatorname{link}(v, N)$, and by the invariance of $\phi$ under simplicial isometries of stars we know that $\phi(v, K)=\phi(v, N)$. Hence (2) is equivalent to

$$
\begin{aligned}
\phi(v, N)= & \Lambda(N)-\frac{1}{2} \sum_{w \in \operatorname{link}(v, N)} \Lambda(\mathcal{O}(v, w) \text {-flap }) \\
& +\frac{1}{2} \Lambda(\text { fan }) f_{1}(\operatorname{link}(v, N))-\Lambda(\text { fan }) \sum_{\sigma^{2} \ni v} \alpha\left(v, \sigma^{2}\right),
\end{aligned}
$$

where the first summation is over all vertices $w$ of $\operatorname{link}(v, N)$, and the second summation is over all 2-simplices of $\operatorname{star}(v, N)$ containing $v$. We will prove (4). 
If $\operatorname{link}(v, N)=\emptyset$, then $N=\{v\}$, and (4) is trivially true, because the DescartesGauss-Bonnet Theorem applied to $N$ yields $\phi(v, N)=\Lambda(N)$, and $\operatorname{link}(v, N)=\varnothing$ implies that all the terms in the right-hand side of (4) other than $\Lambda(N)$ are zero. Hence we suppose that $\operatorname{link}(v, N) \neq \emptyset$.

Let $r$ be a vertex in $\operatorname{link}(v, N)$. Let $M=\operatorname{star}(r, N)$. As remarked after the definition of n-flaps, we know that $M$ is a $\mathcal{O}(v, r)$-flap with end-vertices $v$ and $r$. By the invariance of $\phi$ under simplicial isometries of stars, it follows that $\phi(r, N)=\phi(r, M)$. Hence Step 5 implies that

$$
\phi(r, N)=\phi(r, M)=\frac{1}{2} \Lambda(\mathcal{O}(v, r)-\text { flap })-\Lambda(\text { fan }) \sum_{\sigma^{2} \ni r} \alpha\left(r, \sigma^{2}\right)
$$

where the summation is over all 2-simplices of $M$ containing $r$.

Recall that all the vertices of $N$ other than $v$ are in $\operatorname{link}(v, N)$. Applying the Descartes-Gauss-Bonnet Theorem to $N$ and then solving for $\phi(v, N)$, we see that

$$
\begin{aligned}
\phi(v, N) & =\Lambda(N)-\sum_{w \in \operatorname{link}(v, N)} \phi(w, N) \\
& =\Lambda(N)-\sum_{w \in \operatorname{link}(v, N)}\left\{\frac{1}{2} \Lambda(\mathcal{O}(v, w)-\text { flap })-\Lambda(\text { fan }) \sum_{\sigma^{2} \ni w} \alpha\left(w, \sigma^{2}\right)\right\}
\end{aligned}
$$

by (5)

$$
\begin{gathered}
=\Lambda(N)-\frac{1}{2} \sum_{w \in \operatorname{link}(v, N)} \Lambda(\mathcal{O}(v, w) \text {-flap }) \\
+\sum_{w \in \operatorname{link}(v, N)} \Lambda(\text { fan }) \sum_{\sigma^{2} \ni w} \alpha\left(w, \sigma^{2}\right) \\
=\Lambda(N)-\frac{1}{2} \sum_{w \in \operatorname{link}(v, N)} \Lambda(\mathcal{O}(v, w) \text {-flap }) \\
+\sum_{\sigma^{2} \in \operatorname{star}(v, N)} \Lambda(\text { fan }) \sum_{\substack{y \in \eta^{2} \\
y \neq v}} \alpha\left(y, \sigma^{2}\right)
\end{gathered}
$$

because every 2-simplex in $N$ contains $v$

$$
\begin{gathered}
=\Lambda(N)-\frac{1}{2} \sum_{w \in \operatorname{link}(v, N)} \Lambda(\mathcal{O}(v, w) \text {-flap }) \\
+\sum_{\sigma^{2} \in \operatorname{star}(v, N)} \Lambda(\text { fan })\left[\frac{1}{2}-\alpha\left(v, \sigma^{2}\right)\right]
\end{gathered}
$$

because the sum of the angles in a triangle is $\frac{1}{2}$ 


$$
\begin{aligned}
= & \Lambda(N)-\frac{1}{2} \sum_{w \in \operatorname{link}(v, N)} \Lambda(\mathcal{O}(v, w) \text {-flap }) \\
& +\frac{1}{2} \Lambda(\text { fan }) f_{1}(\operatorname{link}(v, N))-\Lambda(\text { fan }) \sum_{\sigma^{2} \ni v} \alpha\left(v, \sigma^{2}\right),
\end{aligned}
$$

where the last equation holds because $f_{2}(\operatorname{star}(v, N))=f_{1}(\operatorname{link}(v, N))$. Hence (4) holds.

For our next proof, recall the discussion of standard curvature given at the end of Sect. 2.

Proof of Corollary 2.9 Note that every planar fan, every star of every vertex of a simplicial complex in $\mathcal{T}$, and in particular every $\mathcal{O}(v, w)$-flap for appropriate vertices $v$ and $w$, are cones.

Let $K \in \mathcal{T}$, and let $v \in K^{(0)}$. In now follows from Theorem 2.7 that

$$
\phi(v, K)=\Lambda(\text { cone })\left\{1-\frac{1}{2} f_{0}(\operatorname{link}(v, K))+\frac{1}{2} f_{1}(\operatorname{link}(v, K))-\sum_{\sigma^{2} \ni v} \alpha\left(v, \sigma^{2}\right)\right\} .
$$

We also compute the standard curvature of $K$ at $v$, which is given by

$$
\begin{aligned}
S(v, K) & =\sum_{i=0}^{2}(-1)^{i} \sum_{\eta^{i} \ni v} \alpha^{*}\left(v, \eta^{i}\right) \\
& =\alpha^{*}(v, v)-\sum_{\eta^{1} \ni v} \alpha^{*}\left(v, \eta^{1}\right)+\sum_{\eta^{2} \ni v} \alpha^{*}\left(v, \eta^{2}\right) \\
& =1-\sum_{\eta^{1} \ni v} \frac{1}{2}+\sum_{\eta^{2} \ni v}\left[\frac{1}{2}-\alpha\left(v, \eta^{2}\right)\right] \\
& =1-\frac{1}{2} f_{0}(\operatorname{link}(v, K))+\sum_{\eta^{2} \ni v} \frac{1}{2}-\sum_{\eta^{2} \ni v} \alpha\left(v, \eta^{2}\right) \\
& =1-\frac{1}{2} f_{0}(\operatorname{link}(v, K))+\frac{1}{2} f_{1}(\operatorname{link}(v, K))-\sum_{\eta^{2} \ni v} \alpha\left(v, \eta^{2}\right) .
\end{aligned}
$$

It follows that $\phi(v, K)=\Lambda$ (cone) $S(v, K)$. Because $\phi$ satisfies the DescartesGauss-Bonnet Theorem with respect to $\Lambda$ and because standard curvature satisfies the Descartes-Gauss-Bonnet Theorem with respect to the Euler characteristic, it follows that $\Lambda$ equals the Euler characteristic multiplied by $\Lambda$ (cone).

We now turn to the proof of Theorem 2.4. The proof of this theorem is not identical to the proof of Theorem 2.7, because in the former theorem, we assume that $\phi$ is defined only on simplicial surfaces, whereas in the proof of the latter theorem, we make use of various simplicial complexes that are not simplicial surfaces. However, 
we will show how the proof of Theorem 2.7 can be modified to work for the simplicial surface case. (It would be slightly easier to prove Theorem 2.4 if we allowed degenerate simplicial surfaces, but that would add unnecessarily to the hypotheses of the theorem, and so we will not do so.)

Proof of Theorem 2.4 We start with some preliminary observations.

(a) Because all simplicial complexes under consideration are simplicial surfaces, we know that the link of every vertex is a polygonal circle.

(b) Let $K, L \in \mathcal{S}$, and let $v \in K^{(0)}$ and $w \in L^{(0)}$. Suppose that the sum of the angles at $v$ equals the sum of the angles at $w$. Then there are subdivisions $K^{\prime}$ of $K$ and $L^{\prime}$ of $L$ such that $\operatorname{star}\left(v, K^{\prime}\right)$ and $\operatorname{star}\left(w, L^{\prime}\right)$ are simplicially isometric. It then follows as usual that $\phi(v, K)=\phi(w, L)$.

Let $\omega \in(0, \infty)$. Then it is possible to draw a polygonal spiral ribbon $R$ in $\mathbb{R}^{2}$, as in Fig. 1, so that an appropriately chosen bipyramid $B$ on $R$ has angle sum $\omega$ at each of the apices and has angle sum less than 1 at all other vertices (this latter condition will be used later in the proof). Observe that $B \in \mathcal{S}$, because $\mathcal{S}$ contains all bypiramids.

We can therefore think of $\phi$ as a function $(0, \infty) \rightarrow \mathbb{R}$, where for each $\alpha \in$ $(0, \infty)$, we define $\phi(\alpha)$ by $\phi(\alpha)=\phi(v, K)$ for any $K \in \mathcal{S}$ that has a vertex $v$ for which the sum of the angles containing $v$ is $\alpha$.

(c) The continuity of $\phi$ as originally assumed implies that $\phi$ is continuous when thought of as a function $(0, \infty) \rightarrow \mathbb{R}$, as described in Observation (b).

Given the above observations, in order to prove the theorem as originally stated it suffices to show that

$$
\phi(\omega)=\frac{1}{2} \Lambda(\text { pyramid })[1-\omega]
$$

for all $\omega \in(0, \infty)$.

For the rest of the proof, let $\omega \in(0, \infty)$. We have a number of cases.

Case 1 Suppose that $\omega=1$.

Let $T$ be a triangle in $\mathbb{R}^{3}$ with vertices $d_{1}, d_{2}, d_{3}$. Let $\Delta$ be a pyramid on $T$ with apex $b$. For each $i$, let $\delta_{i}$ denote the sum of the angle in $\Delta$ at $d_{i}$, and let $\beta$ denote the sum of the angles in $\Delta$ at $b$. Let $\left\{L_{k}\right\}_{k=1}^{\infty}$ in $\mathbb{R}^{3}$ be a sequence of bipyramids on $T$, where all the $L_{k}$ have $b$ as one of their apices, where the other apex in $L_{k}$ is denoted $e_{k}$ for each $k$, and where the sequence $\left\{e_{k}\right\}_{k=1}^{\infty}$ converges to the centroid of $T$. For each $k$, let $\delta_{i}^{k}$ denote the sum of the angles in $L_{k}$ at $d_{i}$ for each $i$, and let $\gamma^{k}$ denote the sum of the angles in $L_{k}$ at $e_{k}$; observe that the sum of the angles in each $L_{k}$ at $b$ is $\delta$. Clearly $\lim _{k \rightarrow \infty} \delta_{i}^{k}=\delta_{i}$ for all $i$, and $\lim _{k \rightarrow \infty} \gamma^{k}=1=\omega$.

Fig. 1 The set $R$

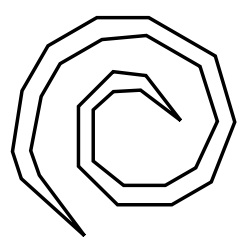


Let $k \in \mathbb{N}$. By applying the Descartes-Gauss-Bonnet Theorem to $L_{k}$, we obtain

$$
\phi\left(b, L_{k}\right)+\sum_{i=1}^{3} \phi\left(d_{i}, L_{k}\right)+\phi\left(e_{k}, L_{k}\right)=\Lambda\left(L_{k}\right) .
$$

Because $\Lambda$ is constant on the set of all pyramids and bipyramids, we know that $\Lambda\left(L_{k}\right)=\Lambda(\Delta)$ for all $k$. Hence

$$
\phi(\beta)+\sum_{i=1}^{3} \phi\left(\delta_{i}^{k}\right)+\phi\left(\gamma^{k}\right)=\Lambda(\Delta) .
$$

Taking the limit as $k \rightarrow \infty$ and using the continuity of $\phi$ as stated in Observation (c), we see that

$$
\phi(\beta)+\sum_{i=1}^{3} \phi\left(\delta_{i}\right)+\phi(\omega)=\Lambda(\Delta) .
$$

On the other hand, applying the Descartes-Gauss-Bonnet Theorem to $\Delta$ yields

$$
\phi(b, \Delta)+\sum_{i=1}^{3} \phi\left(d_{i}, \Delta\right)=\Lambda(\Delta)
$$

which implies

$$
\phi(\beta)+\sum_{i=1}^{3} \phi\left(\delta_{i}\right)=\Lambda(\Delta) .
$$

Comparing (7) with (8) shows that $\phi(\omega)=0$, which is equivalent to (6) when $\omega=1$.

Case 2 Suppose that $\omega=\frac{n-2}{n}$ for some $n \in \mathbb{N}$ such that $n \geq 3$.

Let $S$ and $a_{1}, \ldots, a_{n}$ be as in Step 1 of the proof of Theorem 2.7. Let $\left\{C_{k}\right\}_{k=1}^{\infty}$ in $\mathbb{R}^{3}$ be a sequence of pyramids on $S$, where the apex of $C_{k}$ is denoted $x_{k}$ for each $k$, where the 2-simplices of $C_{k}$ containing $x_{k}$ are all congruent isosceles triangles, and where the sequence $\left\{x_{k}\right\}_{k=1}^{\infty}$ converges to the centroid of $S$. For each $k$, let $\omega_{i}^{k}$ denote the sum of the angles in $C_{k}$ at $a_{i}$, and let $\beta^{k}$ denote the sum of the angles in $C_{k}$ at $x_{k}$. Then $\lim _{k \rightarrow \infty} \omega_{i}^{k}=\omega$ for all $i$, and $\lim _{k \rightarrow \infty} \beta^{k}=1$.

We now proceed similarly to Case 1 . Let $k \in \mathbb{N}$. By applying the Descartes-GaussBonnet Theorem to $C_{k}$ we obtain

$$
\sum_{i=1}^{n} \phi\left(\omega_{i}^{k}\right)+\phi\left(\beta^{k}\right)=\Lambda\left(C_{k}\right)
$$

By construction we know that the $\omega_{i}^{k}$ are all equal to each other, and hence

$$
\phi\left(\omega_{1}^{k}\right)=\frac{1}{n}\left[\Lambda(\text { pyramid })-\phi\left(\beta^{k}\right)\right] .
$$


Taking the limit as $k \rightarrow \infty$ and using the continuity of $\phi$, as well as Case 1 , we see that

$$
\phi(\omega)=\frac{1}{n}[\Lambda(\text { pyramid })-\phi(1)]=\frac{1}{n} \Lambda(\text { pyramid }) .
$$

Because $\omega=\frac{n-2}{n}$, we have $n=\frac{2}{1-\omega}$, and substituting this formula for $n$ into (9) yields (6).

Case 3 Suppose that $\omega$ is a rational number such that $0<\omega<1$.

There are $p, q \in \mathbb{N}$ such that $\omega=\frac{2 p}{q}$. Because $\omega<1$, we have $q \geq 3$. The proof in this case is similar to that of Case 2 , except that we use pyramids on the polygon $D$ from Step 2 of the proof of Theorem 2.7; we omit the details.

Case 4 Suppose that $\omega \in(0,1)$.

Equation (6) follows immediately from Case 3 and the continuity of $\phi$.

Case 5 Suppose that $\omega \in[1, \infty)$.

As remarked in Observation (b), it is possible to draw a polygonal spiral ribbon $R$ in $\mathbb{R}^{2}$ so that an appropriately chosen bipyramid $B$ on $R$ has angle sum $\omega$ at each of its apices and has angle sum less than 1 at all other vertices. Suppose that the vertices of $R$ are denoted $b_{1}, \ldots, b_{m}$, and the apices of $B$ are denoted $x$ and $y$. For each $i$, let $\beta_{i}$ denote the sum of the angles in $B$ at $b_{i}$. By Case 4 we know that $\phi\left(\beta_{i}\right)=\frac{1}{2} \Lambda$ (pyramid) $\left[1-\beta_{i}\right]$ for each $i$.

The classical angle defect at $b_{i}$ is $1-\beta_{i}$, and the classical angle defect at each of $x$ and $y$ is $1-\omega$. Using the Descartes-Gauss-Bonnet Theorem for the classical angle defect applied to $B$, we have

$$
2=\chi(B)=\sum_{i=1}^{m}\left[1-\beta_{i}\right]+2[1-\omega],
$$

which implies that

$$
\omega=\frac{1}{2} \sum_{i=1}^{m}\left[1-\beta_{i}\right] .
$$

Applying the Descartes-Gauss-Bonnet Theorem for $\phi$ to $B$ yields

$$
\Lambda(B)=\sum_{i=1}^{m} \phi\left(b_{i}, B\right)+\phi(x, B)+\phi(y, B),
$$

and using arguments similar to those used previously in this proof, we deduce that

$$
\begin{aligned}
\phi(\omega) & \left.=\frac{1}{2}\{\Lambda \text { (pyramid })-\sum_{i=1}^{m} \phi\left(\beta_{i}\right)\right\} \\
& =\frac{1}{2} \Lambda \text { (pyramid) }\left\{1-\frac{1}{2} \sum_{i=1}^{m}\left[1-\beta_{i}\right]\right\}=\frac{1}{2} \Lambda(\text { pyramid })[1-\omega] .
\end{aligned}
$$


Acknowledgements I would like to thank Tom Banchoff and the referees for very helpful suggestions.

\section{References}

1. Banchoff, T.: Critical points and curvature for embedded polyhedra. J. Differ. Geom. 1, 245-256 (1967)

2. Banchoff, T.: Critical points and curvature for embedded polyhedra, II. Prog. Math. 32, 34-55 (1983)

3. Bloch, E.D.: The angle defect for arbitrary polyhedra. Beitr. Algebra Geom. 39, 379-393 (1998)

4. Bloch, E.D.: Critical points and the angle defect. Geom. Dedicata 109, 121-137 (2004)

5. Bloch, E.D.: The angle defect for odd-dimensional simplicial manifolds. Discrete Comput. Geom. 35, 311-328 (2006)

6. Budach, L.: Lipschitz-Killing curvatures of angular partially ordered sets. Adv. Math. 78, 140-167 (1989)

7. Cheeger, J.: Spectral geometry of singular Riemannian spaces. J. Differ. Geom. 18, 575-657 (1983)

8. Cheeger, J., Muller, W., Schrader, R.: On the curvature of piecewise flat spaces. Commun. Math. Phys. 92, 405-454 (1984)

9. Federico, P.J.: Descartes on Polyhedra. Springer, New York (1982)

10. Forman, R.: The Euler characteristic is the unique locally determined numerical invariant of finite simplicial complexes which assigns the same number to every cone. Discrete Comput. Geom. 23(4), 485-488 (2000)

11. Forman, R.: Bochner's method for cell complexes and combinatorial Ricci curvature. Discrete Comput. Geom. 29(3), 323-374 (2003)

12. Glaser, L.C.: Geometrical Combinatorial Topology, vols. I-II. Van Nostrand Reinhold, New York (1970)

13. Grünbaum, B.: Convex Polytopes. Wiley, New York (1967)

14. Grünbaum, B.: Grassmann angles of convex polytopes. Acta. Math. 121, 293-302 (1968)

15. Grünbaum, B., Shephard, G.C.: Descartes' theorem in $n$ dimensions. Enseign. Math. 37(2), 11-15 (1991)

16. Hudson, J.F.P.: Piecewise Linear Topology. Benjamin, Menlo Park (1969)

17. Levitt, N.: The Euler characteristic is the unique locally determined numerical homotopy invariant of finite complexes. Discrete Comput. Geom. 7, 59-67 (1992)

18. McMullen, P.: Non-linear angle-sum relations for polyhedral cones and polytopes. Math. Proc. Camb. Philos. Soc. 78, 247-261 (1975)

19. Perles, M.A., Shephard, G.C.: Angle sums of convex polytopes. Math. Scand. 21, 199-218 (1967)

20. Shephard, G.C.: An elementary proof of Gram's Theorem for convex polytopes. Can. J. Math. 19, 1214-1217 (1967)

21. Shephard, G.C.: Angle deficiencies of convex polytopes. J. Lond. Math. Soc. 43, 325-336 (1968)

22. Wintgen, P.: Normal cycle and integral curvature for polyhedra in Riemannian manifolds. In: Soós, G., Szenthe, J. (eds.) Differential Geometry. Colloq. Math. Soc. János Bolyai, vol. 31, pp. 805-816. North-Holland, Amsterdam (1982)

23. Zähle, M.: Approximation and characterization of generalized Lipschitz-Killing curvatures. Ann. Glob. Anal. Geom. 8, 249-260 (1990) 